\title{
On a Five-Dimensional Chaotic System Arising from Double-Diffusive Convection in a Fluid Layer
}

\author{
R. Idris, ${ }^{1}$ Z. Siri, ${ }^{2}$ and I. Hashim ${ }^{3}$ \\ ${ }^{1}$ Department of Mathematics, Faculty of Science and Technology, Universiti Malaysia Terengganu, 21030 Kuala Terengganu, \\ Terengganu, Malaysia \\ ${ }^{2}$ Institute of Mathematical Sciences, University of Malaya, 50603 Kuala Lumpur, Malaysia \\ ${ }^{3}$ School of Mathematical Sciences, Universiti Kebangsaan Malaysia, 43600 Bangi, Selangor, Malaysia
}

Correspondence should be addressed to I. Hashim; ishak_h@ukm.my

Received 19 November 2012; Accepted 14 January 2013

Academic Editor: Patricia J. Y. Wong

Copyright @ 2013 R. Idris et al. This is an open access article distributed under the Creative Commons Attribution License, which permits unrestricted use, distribution, and reproduction in any medium, provided the original work is properly cited.

A chaotic system arising from double-diffusive convection in a fluid layer is investigated in this paper based on the theory of dynamical systems. A five-dimensional model of chaotic system is obtained using the Galerkin truncated approximation. The results showed that the transition from steady convection to chaos via a Hopf bifurcation produced a limit cycle which may be associated with a homoclinic explosion at a slightly subcritical value of the Rayleigh number.

\section{Introduction}

The concept of sensitivity on initial conditions where a small difference on initial conditions may produce large variations in the long-term behaviour of the system is pivotal in chaos theory. This behaviour is also known as the "butterfly effect" related to work done by Lorenz [1] where it is already described by Henri Poincare in 1890 in the literature in a particular case of the three-body problem. Chaotic behaviour has been studied intensively in various dynamical systems; see, for example, [2-14].

The investigation of free convection in the RayleighBénard problem is receiving much attention due to its wide application in different disciplines such as biotechnology for the description of the convection with the microorganisms diffusion, in astrophysics for simulation of the influence of the helium diffusion on convective motions in the stars, in oceanography for the investigation of the salinity influence on the convective motions in the seas, and in engineering and geology. Research in double-diffusive convection begins after the work done by sea-going oceanographers in order to measure the fluctuation of temperature and salinity as a function of depth as stated in the paper of Huppert and Turner [15]. Then, Knobloch et al. [16] and Bhattacharjee [17] studied the transition to chaos in double-diffusive convection with stressfree boundary conditions where oscillatory solution exists. They showed that the instability of fluid becomes oscillatory when thermal Rayleigh number is raised and the truncated model suggests that the appearance of chaos is associated with heteroclinic bifurcations.

Two-dimensional thermosolutal convection between free boundaries was studied numerically by Veronis [18]. From their observation, they found that when the solutal Rayleigh number is large enough, the oscillations underwent a bifurcation to asymmetry as thermal Rayleigh number increased and, for the larger values of solutal Rayleigh number, the transition from chaos to steady motion occurs.

Sibgatullin et al. [19] studied some properties of twodimensional stochastic regimes of double-diffusive convection in a plane layer. Using the Bubnov-Galerkin method, they obtained that, with the growth of Rayleigh numbers of heat and salinity, the structure of one-dimensional curve becomes more irregular and sophisticated. Transition to chaos in double-diffusive Marangoni convection was studied by $\mathrm{Li}$ et al. [20]. It was found that the supercritical solution branch takes a quasiperiodicity and phase locking route to chaos while the subcritical branch follows the RuelleTakens-Newhouse scenario. The transitions from regular to 
chaotic dynamics and analysis of the hyper, hyper-hyper, and spatial-temporal chaos using the Lyapunov exponents of continuous mechanical systems have been studied in [21$24]$; they found the Sharkovskii windows of periodicity in the systems investigated.

The objective of the present paper is to study the weak turbulence and chaos in double-diffusive convection involving temperature and concentration as the thermal Rayleigh number increases with rigid, no-slip horizontal boundary condition. Applying the truncated Galerkin approximation to the governing equations yields an autonomous system with five ordinary differential equations which can be used to understand low-dimensional dynamics before moving to studying more complex systems.

\section{Mathematical Formulation}

Consider a two-dimensional layer of fluid of depth $H$ subject to gravity and heated from below as shown in Figure 1. A Cartesian coordinate system is used such that the vertical axis $z$ is collinear with gravity, that is, $\widehat{\mathbf{e}}_{\mathbf{g}}=-\widehat{\mathbf{e}}_{\mathbf{z}}$. The two long walls are maintained at temperatures $T_{H}$ and $T_{C}$ and solute concentrations $S_{H}$ and $S_{C}$, respectively. A relationship between density, temperature, and solute concentration is assumed linear and can be presented by the following form $\rho=\rho_{0}\left[1-\alpha\left(T-T_{C}\right)+\alpha_{s}\left(S-S_{C}\right)\right]$, where $\alpha$ and $\alpha_{s}$ are volume expansion coefficients due to variations of thermal and solute concentrations. The Boussinesq approximation is applied for the effects of density variations for the gravity term in momentum equation. Therefore, the set of equations governing the conservation of mass, momentum, energy, and concentration for fluid flow is given by the following:

$$
\begin{gathered}
\nabla \cdot \mathbf{V}=0 \\
\rho_{0}\left[\frac{\partial \mathbf{V}}{\partial t}+\mathbf{V} \cdot \mathbf{V}\right]=-\nabla p+\nabla^{2} \mathbf{V}+\rho \widehat{\mathbf{e}}_{\mathbf{z}} \\
\frac{\partial T}{\partial t}+\mathbf{V} \cdot \nabla T=\eta \nabla^{2} T \\
\frac{\partial S}{\partial t}+\mathbf{V} \cdot \nabla S=\eta_{s} \nabla^{2} S
\end{gathered}
$$

We nondimensionalize (1)-(4) using the following transformations:

$$
\begin{gathered}
\mathbf{V}_{*}=\frac{H_{*}}{\eta_{*}} \mathbf{V}, \quad p_{*}=\frac{H_{*}^{2}}{\rho_{0} \eta_{*}^{2}} p, \\
T_{*}=\frac{\left(T-T_{C}\right)}{\Delta T_{c}}, \quad S_{*}=\frac{\left(S-S_{C}\right)}{\Delta S_{c}}, \\
\left(x_{*}, y_{*}, z_{*}\right)=H_{*}(x, y, z), \quad t_{*}=\frac{t H_{*}^{2}}{\eta_{*}},
\end{gathered}
$$

where $\mathbf{V}_{*}=\left(u_{*}, v_{*}, w_{*}\right)$ is the velocity component, $p_{*}$ is the pressure, $\left(T-T_{C}\right)$ and $\left(S-S_{C}\right)$ are the temperature and solute concentration variations, $\eta_{*}$ is the effective thermal diffusivity, and $v_{*}$ is fluid's viscosity.

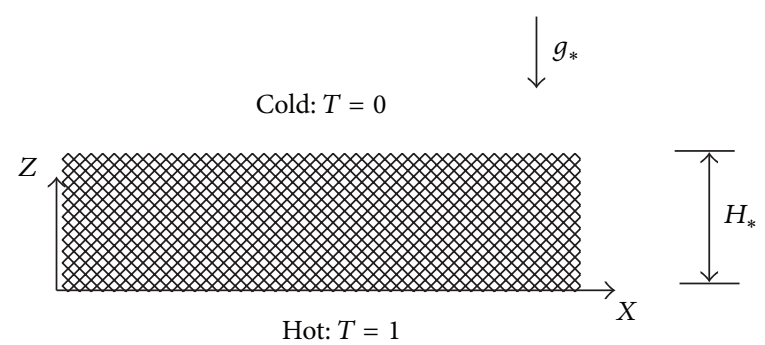

FIgURe 1: Physical model.

In this model, all the boundaries are rigid and the solution must follow the impermeability conditions there, that is, $\mathbf{V} \cdot \widehat{\mathbf{e}}_{\mathbf{n}}=0$ on the boundaries, where $\widehat{\mathbf{e}}_{\mathbf{n}}$ is a unit vector normal to the boundary. The temperature and solute concentration boundary conditions are $T=S=1$ at $z=0$ and $T=S=0$ at $z=1$.

For convective rolls having axes parallel to the shorter dimension (i.e., $y=0) v=0$, by applying the curl operator on (2) to eliminate the pressure and introducing the stream function defined by $u=\partial \psi / \partial z$ and $w=-\partial \psi / \partial x$, we get

$$
\begin{gathered}
{\left[\frac{1}{\operatorname{Pr}}\left(\frac{\partial}{\partial t}+\frac{\partial \psi}{\partial z} \frac{\partial}{\partial x}-\frac{\partial \psi}{\partial x} \frac{\partial}{\partial z}\right)-\nabla^{2}\right] \nabla^{2} \psi=-\operatorname{Ra} \frac{\partial T}{\partial x}+R_{s} \frac{\partial S}{\partial x}} \\
\frac{\partial T}{\partial t}+\frac{\partial \psi}{\partial z} \frac{\partial T}{\partial x}-\frac{\partial \psi}{\partial x} \frac{\partial T}{\partial z}=\frac{\partial^{2} T}{\partial x^{2}}+\frac{\partial^{2} T}{\partial z^{2}} \\
\frac{\partial S}{\partial t}+\frac{\partial \psi}{\partial z} \frac{\partial S}{\partial x}-\frac{\partial \psi}{\partial x} \frac{\partial S}{\partial z}=\frac{1}{\mathrm{Le}}\left(\frac{\partial^{2} S}{\partial x^{2}}+\frac{\partial^{2} S}{\partial z^{2}}\right)
\end{gathered}
$$

where

$$
\begin{gathered}
\operatorname{Pr}=\frac{\nu_{*}}{\eta_{*}}, \quad \mathrm{Ra}=\frac{\alpha_{*} \Delta T_{c} g_{*} H_{*}^{3}}{\eta_{*} \nu_{*}}, \\
R_{s}=\frac{\alpha_{s *} \Delta S_{c} g_{*} H_{*}^{3}}{\eta_{*} \nu_{*}}, \quad \mathrm{Le}=\frac{\eta_{*}}{\eta_{s *}}
\end{gathered}
$$

which are, respectively, the Prandtl number, the Rayleigh number, the solutal Rayleigh number, and the Lewis number. The boundary conditions for the stream function are $\psi=0$ on the horizontal boundaries. Equation (6) forms a nonlinear coupled system which together with the corresponding boundary conditions allows for a basic motionless conduction solution.

\section{Diminished Set of Equation}

In order to obtain the solution to (6), we represent the stream function, temperature, and solutal distributions in the following form:

$$
\begin{gathered}
\psi=A_{11} \sin (\kappa x) \sin (\pi z), \\
T=1-z+B_{11} \cos (\kappa x) \sin (\pi z)+B_{02} \sin (2 \pi z), \\
S=1-z+C_{11} \cos (\kappa x) \sin (\pi z)+C_{02} \sin (2 \pi z) .
\end{gathered}
$$


Substituting (8) into (6), multiplying the equations by the orthogonal eigenfunctions corresponding to (8), and then integrating them over the spatial domain yield a set of five ordinary differential equations for the time evolution of the following amplitudes:

$$
\begin{gathered}
\frac{d \bar{A}_{11}}{d \tau}=\operatorname{Pr}\left[\bar{B}_{11}-\bar{A}_{11}+\bar{C}_{11}\right], \\
\frac{d \bar{B}_{11}}{d \tau}=-\bar{B}_{11}+R \bar{A}_{11}-\bar{A}_{11} \bar{B}_{02}, \\
\frac{d \bar{B}_{02}}{d \tau}=\bar{A}_{11} \bar{B}_{11}-\lambda \bar{B}_{02}, \\
\frac{d \bar{C}_{11}}{d \tau}=-\frac{\bar{C}_{11}}{\operatorname{Le}}+R_{s} \bar{A}_{11}-\bar{A}_{11} \bar{C}_{02}, \\
\frac{d \bar{C}_{02}}{d \tau}=\bar{A}_{11} \bar{C}_{11}-\frac{\lambda}{\mathrm{Le}} \bar{C}_{02} .
\end{gathered}
$$

In (9), the time, the amplitudes, the Rayleigh number, and the solutal Rayleigh number were rescaled, and the following notations are introduced as follows:

$$
\begin{gathered}
\bar{A}_{11}=\frac{\left(\kappa / \kappa_{\mathrm{cr}}\right)}{\left[\left(\kappa / \kappa_{\mathrm{cr}}\right)^{2}+2\right]} A_{11}, \quad \bar{B}_{11}=\kappa_{\mathrm{cr}} R B_{11}, \\
\bar{B}_{02}=\pi R B_{02}, \quad \bar{C}_{11}=\kappa_{\mathrm{cr}} R_{s} C_{11}, \\
\bar{C}_{02}=\pi R_{s} C_{02}, \quad R=\frac{\mathrm{Ra}}{\mathrm{Ra}_{c}}, \quad R_{s}=\frac{R_{s}}{R_{s c}}, \\
\tau=\left(\kappa^{2}+\pi^{2}\right) t, \quad \lambda=\frac{8}{\left[\left(\kappa / \kappa_{\mathrm{cr}}\right)^{2}+2\right]}, \\
\mathrm{Ra}_{c}=R_{s c}=\frac{\left(\kappa^{2}+\pi^{2}\right)^{3}}{\kappa^{2}}, \quad \kappa_{\mathrm{cr}}=\frac{\pi}{\sqrt{2}} .
\end{gathered}
$$

Rescaling the equation again in the forms

$$
\begin{gathered}
X=\frac{\bar{A}_{11}}{\sqrt{\lambda(R-1)}}, \quad Y=\frac{\bar{B}_{11}}{\sqrt{\lambda(R-1)}}, \quad Z=\frac{\bar{B}_{02}}{(R-1)}, \\
U=\frac{\bar{C}_{11}}{\sqrt{\lambda(R-1)}}, \quad W=\frac{\bar{C}_{02}}{(R-1)},
\end{gathered}
$$

gives the following set of scaled equations which are equivalent to (9):

$$
\begin{gathered}
\dot{X}=\operatorname{Pr}(Y-X-U), \\
\dot{Y}=R X-Y-(R-1) X Z, \\
\dot{Z}=\lambda(X Y-Z), \\
\dot{U}=R_{s} X-\frac{U}{\mathrm{Le}}-(R-1) X W, \\
\dot{W}=\lambda\left(X U-\frac{W}{\mathrm{Le}}\right),
\end{gathered}
$$

\section{Linear Stability Analysis}

In this paper, we investigate the chaotic behaviour with low Prandtl number in double-diffusive convection. We obtained system (12) that provides a set of nonlinear equations with five parameters. The value of $\lambda$ has to be consistent with the wave number at the convection threshold, a requirement for the convection cells to fit into the domain and fulfill the boundary conditions. However, the Lorenz equations have been extensively analyzed and solved for parameter values corresponding to convection in pure fluids and, even there, the parameter values most regularly used correspond to $\operatorname{Pr}=10$ and $\lambda=8 / 3$. Therefore, it is of interest to analyze and solve the corresponding equations for parameter values corresponding to the problem under investigation. We employ the MATLAB ODE45 routine for obtaining the numerical solutions.

Before attempting the numerical solution of system (12), it is useful to examine the local stability of equilibrium points. System (12) has the three basic properties which we will discuss in the following: dissipation, fixed points, and stability of fixed points.

4.1. Dissipation. System (12) is dissipative since

$$
\begin{aligned}
\nabla \cdot \widehat{V} & =\frac{\partial \dot{X}}{\partial X}+\frac{\partial \dot{Y}}{\partial Y}+\frac{\partial \dot{Z}}{\partial Z}+\frac{\partial \dot{U}}{\partial U}+\frac{\partial \dot{W}}{\partial W} \\
& =-\left(1+\operatorname{Pr}+\frac{1+\lambda}{\mathrm{Le}}+\lambda\right) \\
& <0 .
\end{aligned}
$$

Therefore, if the set of initial points in the phase space occupies region $\widehat{V}(0)$ at $\tau=0$, then, after some time, $\tau$, the endpoints of the corresponding trajectories will fill a volume

$$
\widehat{V}(\tau)=\widehat{V}(0) \exp \left[-\left(1+\operatorname{Pr}+\frac{(1+\lambda)}{\operatorname{Le}}+\lambda\right)\right] .
$$

The expression indicates that the volume decreases monotonically with time.

4.2. Fixed Points. The fixed points for velocity, temperature, and solute concentration can be obtained by setting the derivatives of system (12) to zero:

$$
\begin{gathered}
\operatorname{Pr}(Y-X-U)=0, \\
R X-Y-(R-1) X Z=0, \\
\lambda(X Y-Z)=0, \\
R_{s} X-\frac{U}{\mathrm{Le}}-(R-1) X W=0, \\
\lambda\left(X U-\frac{W}{\mathrm{Le}}\right)=0 .
\end{gathered}
$$

There is one trivial solution, that is, the origin in the phase space

$$
X_{1}=Y_{1}=Z_{1}=U_{1}=W_{1}=0,
$$


which corresponds to the motionless solution. The other nonzero fixed points are given by the following:

$$
\begin{aligned}
& X_{2,3}= \pm \frac{h_{2}}{\sqrt{2}}, \\
& Y_{2,3}= \pm \frac{\left(-1+\operatorname{Le}^{2}(R+1)-\mathrm{Le}_{s}+h_{1}\right) X_{2,3}}{2\left(\mathrm{Le}^{2}-1\right)}, \\
& Z_{2,3}=\frac{\left(1-2 R+\operatorname{Le}^{2}(R-1)+\mathrm{Le}_{s}-h_{1}\right)}{2\left(\mathrm{Le}^{2}-1\right)(R-1)}, \\
& U_{2,3}= \pm \frac{\left(1+\operatorname{Le}^{2}(R-1)-\mathrm{Le}_{s}+h_{1}\right) X_{2,3}}{2\left(\mathrm{Le}^{2}-1\right)}, \\
& W_{2,3}=-\frac{\left(1+\mathrm{Le}^{2}(R-1)+\mathrm{Le}_{s}-2 \mathrm{Le}^{3} R_{s}+h_{1}\right)}{2 \operatorname{Le}\left(\mathrm{Le}^{2}-1\right)(R-1)}, \\
& X_{4,5}= \pm \frac{h_{3}}{\sqrt{2}} \\
& Y_{4,5}= \pm \frac{\left(-1+\operatorname{Le}^{2}(R+1)-\mathrm{Le}_{s}-h_{1}\right) X_{4,5}}{2\left(\mathrm{Le}^{2}-1\right)}, \\
& Z_{4,5}=\frac{\left(1-2 R+\operatorname{Le}^{2}(R-1)+\mathrm{Le} R_{s}+h_{1}\right)}{2\left(\mathrm{Le}^{2}-1\right)(R-1)}, \\
& U_{4,5}= \pm \frac{\left(1+\operatorname{Le}^{2}(R-1)-\mathrm{Le}_{s}-h_{1}\right) X_{4,5}}{2\left(\mathrm{Le}^{2}-1\right)}, \\
& W_{4,5}=\frac{\left(-1+\mathrm{Le}^{2}(1-R)-\mathrm{Le}_{s}+2 \mathrm{Le}^{3} R_{s}+h_{1}\right)}{2 \mathrm{Le}\left(\mathrm{Le}^{2}-1\right)(R-1)},
\end{aligned}
$$

where

$$
\begin{gathered}
h_{1} \\
=\sqrt{\left(\left(1+\operatorname{Le}^{2}(R-1)\right)^{2}-2 \operatorname{Le}_{s}\left(\operatorname{Le}^{2}(R+1)-1\right)+\operatorname{Le}^{2} R_{s}^{2}\right)}, \\
h_{2}=\sqrt{-\frac{\left(1+\operatorname{Le}^{2}(1-R)+\operatorname{Le} R_{s}+h_{1}\right)}{\operatorname{Le}^{2}(R-1)}}, \\
h_{3}=\sqrt{\frac{\left(-1+\operatorname{Le}^{2}(R-1)-\operatorname{Le}_{s}+h_{1}\right)}{\operatorname{Le}^{2}(R-1)}} .
\end{gathered}
$$

The system has five fixed points. When $R=0$, the five fixed points are all real. Thus, when $(R-1)>0, h_{1}$ is always real and $h_{2}$ and $h_{3}$ are always complex; therefore, the three fixed points $\left(X_{i}, Y_{i}, U_{i}\right)$ are all complex and the other two fixed points, $\left(Z_{i}, W_{i}\right)$, are all real for $i=2, \ldots, 5$. The fixed point $\left(X_{1}, Y_{1}, Z_{1}, U_{1}, W_{1}\right)$ corresponds to motionless solution and $\left(X_{i}, Y_{i}, Z_{i}, U_{i}, W_{i}\right)$, where $i=2, \ldots, 5$ corresponds to the convective solution.
4.3. Stability of the Fixed Points. The Jacobian matrix of (12) can be written as follows:

$$
J=\left[\begin{array}{ccccc}
-\operatorname{Pr} & \operatorname{Pr} & 0 & -\operatorname{Pr} & 0 \\
R-(R-1) Z & -1 & -(R-1) X & 0 & 0 \\
\lambda Y & \lambda X & -\lambda & 0 & 0 \\
R_{s}-(R-1) W & 0 & 0 & -\frac{1}{\mathrm{Le}} & (R-1) X \\
\lambda U & 0 & 0 & \lambda X & -\frac{\lambda}{\mathrm{Le}}
\end{array}\right] .
$$

Since the matrix is $5 \times 5$, it is hard to obtain the eigenvalues in a closed form. Hence, the numerical calculation can be performed to discuss the stability at the fixed point. The motionless solution loses stability and the convection solution takes over at the fixed point $\left\{X_{1}, Y_{1}, Z_{1}, U_{1}, W_{1}\right\}$ with the critical value, $R_{c 1}$. Numerical results for the value of $R_{c 1}$, which corresponds to the onset of convection, is obtained for various values of Le and $R_{s}$ with the value of parameters $\operatorname{Pr}=10$ and $\lambda=8 / 3$ as shown in Figure 2. Increasing the values of Le and $R_{s}$ increases the value of $R_{c 1}$.

The stability of the fixed points $\left(X_{i}, Y_{i}, Z_{i}, U_{i}, W_{i}\right)$ $(i=2, \ldots, 5)$ is associated with the convective solution. The evolution of the complex eigenvalues of $J$ in the case $\operatorname{Pr}=10, \lambda=8 / 3$, and Le $=0.1$ is plotted as shown in Figure 3(a) for the fixed point $\left(X_{4,5}, Y_{4,5}, Z_{4,5}, U_{4,5}, W_{4,5}\right)$. These two roots become a complex conjugate at $R \simeq 3.32,3.97,4.63$, and 5.28 for the case $R_{s}=15,20,25$, and 30, respectively. At these points exactly, they still have negative real parts; therefore, the convection fixed points are stable, that is, spiral nodes. Of all the cases, both the imaginary and real parts of these two complex conjugate eigenvalues increase as $R$ increases and they cross the imaginary axis on the complex plane, so as a result their real part becomes nonnegative at a value of $R_{c 2} \simeq 46.37,57.34,69.33,82.18$. At these points, the convective fixed points lose their stability and another periodic, quasiperiodic or chaotic solution takes over. Figure 3(b) shows the evolution of the complex eigenvalues of $J$ for the case $\operatorname{Pr}=10, \lambda=8 / 3$ and $R_{s}$ for the fixed point $\left(X_{4,5}, Y_{4,5}, Z_{4,5}, U_{4,5}, W_{4,5}\right)$. These two roots become a complex conjugate at $R \simeq 3.33,5.23,7.03,8.73$ for the case of Le $=0.1,0.2,0.3,0.4$, respectively. As mentioned before, the convection fixed points are stable and become spiral nodes. Their real part becomes nonnegative at a value of $R_{c 2} \simeq 31.71,34.96,40.05,46.37$. Therefore, at these points, the convective fixed points lose their stability and another periodic, quasiperiodic, or chaotic solution takes over.

While for the fixed point $\left(X_{2,3}, Y_{2,3}, Z_{2,3}, U_{2,3}, W_{2,3}\right)$, the evolution of the complex eigenvalues of $J$ with the same parameter values is always on the positive side of $\operatorname{Re}(\Lambda)$, does not cross the zero axis for $\operatorname{Re}(\Lambda)$, and is not of interest in this study. 


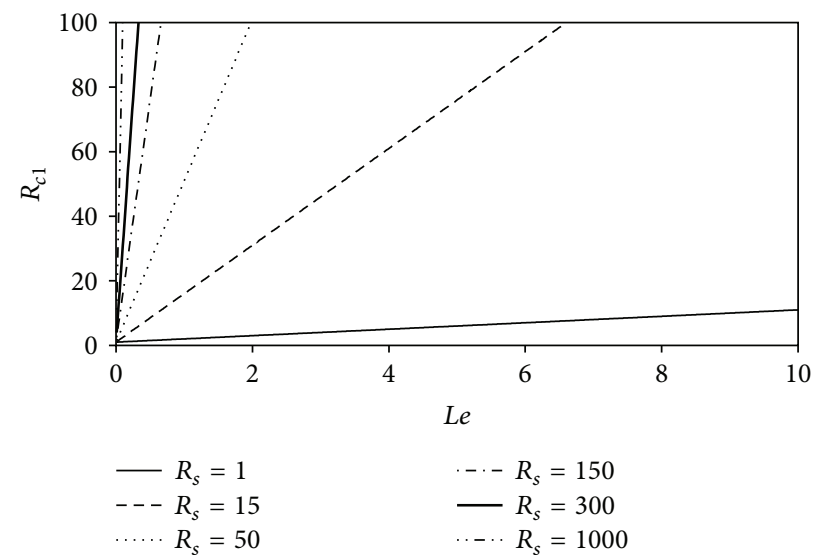

(a)

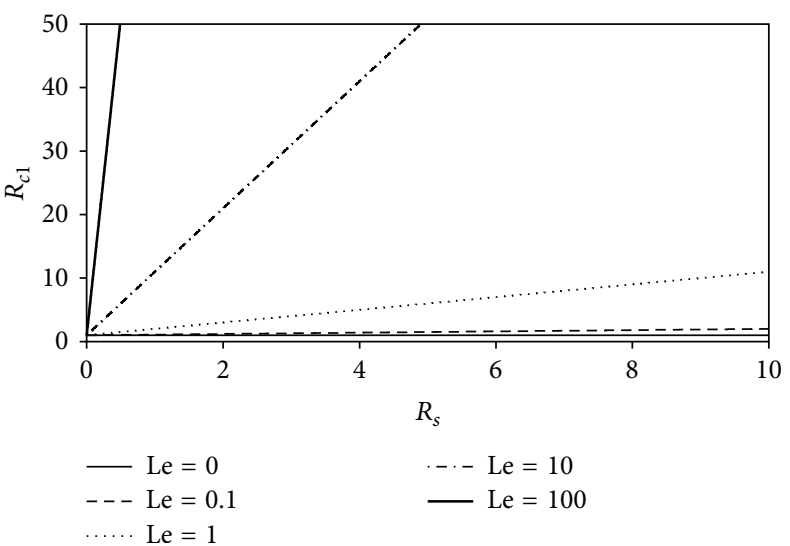

(b)

FIgURE 2: The critical Rayleigh number $R_{c 1}$ as function of (a) Le and (b) $R_{s}$.

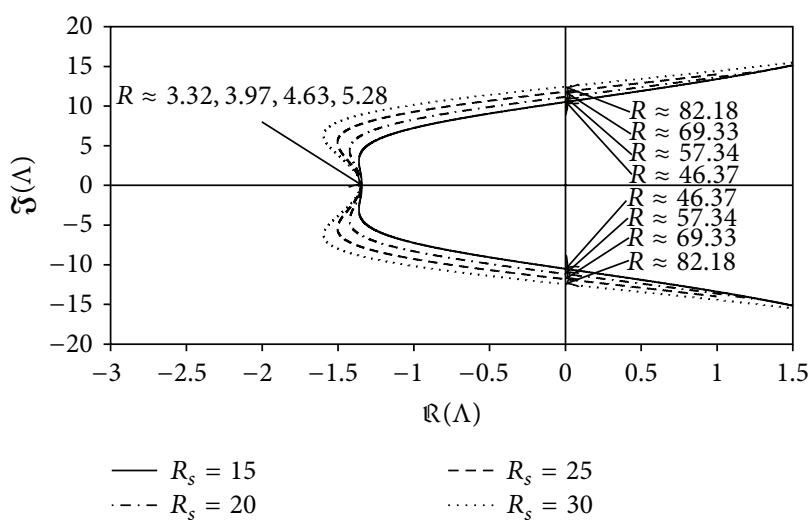

(a)

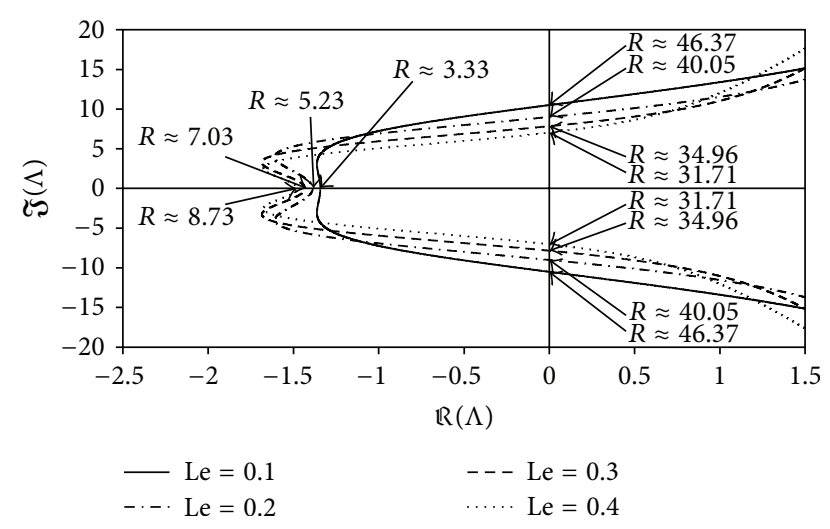

(b)

Figure 3: The evolution of the complex eigenvalues with increasing Rayleigh number for $\operatorname{Pr}=10, \lambda=8 / 3$, (a) $\mathrm{Le}=0.1$, and $(\mathrm{b}) R_{s}=15$.

\section{Results and Discussion}

5.1. Bifurcations and Transition to Chaos. In this study, we focused on the dynamic behaviour of thermal convection in double-diffusive fluid layer. The values of $\operatorname{Pr}$ and $\lambda$ used in all computations are 10 and $8 / 3$, respectively, which are consistent with the critical Rayleigh number $\left(R_{c} \simeq 24.74\right)$ and the critical wave number at marginal stability in fluid layer convection. All solutions were obtained using the same initial conditions, which were selected to be in the neighborhood of the positive convection fixed point. The initial conditions are at $\tau=0: X, Y, Z, U, W=0.9$. All computations were carried out with the value of maximum time, $\tau_{\max }=210$, and a step size $\Delta \tau=0.001$ using the built-in ODE45 method in MATLAB R2010a.

The bifurcation diagrams illustrated in Figure 4 show the peaks and valleys in the posttransient values of $Z$ versus $R$. In Figure $4(\mathrm{a})$, for $0<R<46.37$ we have one-point attractors, but the "attracted" value of $Z$ increases as $R$ increases, at least to $R \simeq 46.37$. Bifurcation occurs at $R \simeq 46.37,48$ until just beyond $R=50$, where the system is chaotic. However, the system is not chaotic for all values of $R>50$, and we will discuss it using phase-portrait diagram. When we fix Le $=0.1$ and increase $R_{s}$ from 15 to 30 , the range of onepoint attractor changes to $0<R<82.31$; this is shown in Figure 4(b), while in Figure 4(c), one-point attractor dropped to $0<R<31.86$ in the case of Le $=0.4$ and $R_{s}=15$. Here we can conclude that increasing the value of the solutal Rayleigh number (with fixed value of Lewis number) will delay the convection process. But increasing the value of Lewis number (with fixed value of solutal Rayleigh number) will enhance the onset of chaos.

Figure 5 shows the projections of the trajectory's data points on the $X-Y-Z$ plane for Le $=0.1$ and $R_{s}=15$. From Figure 5(a), we obtain a solitary limit cycle signifying the loss of stability of the steady convective fixed points. The subcritical value for this transition is $R_{c 2}=46.37$. Figure 5(b) shows the projections of the trajectory's data points for $R=48$. At this point, the homoclinic explosion occurs and the chaotic regime with the strange attractor takes over. The homoclinic explosion behaviour giving birth to a stable periodic orbit with period- 8 at $R=250$ is presented 


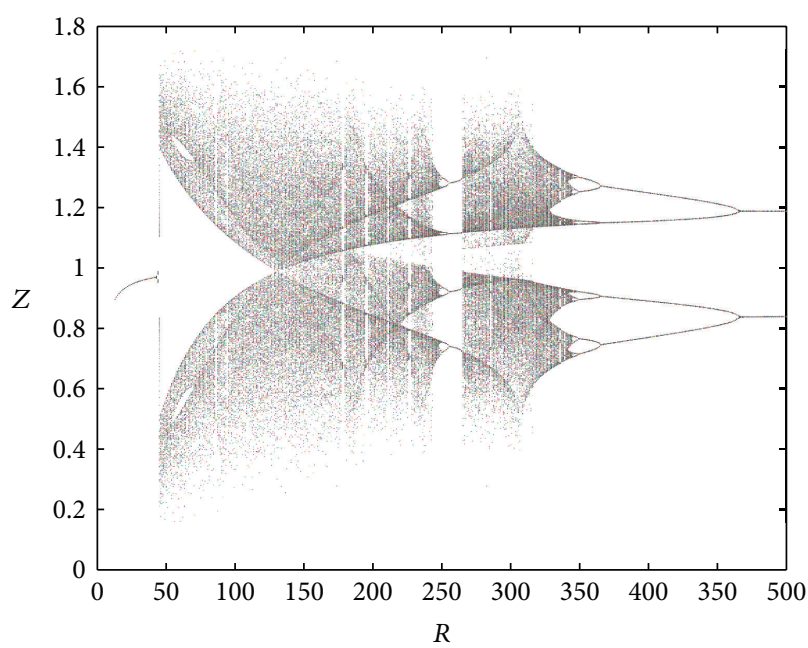

(a)

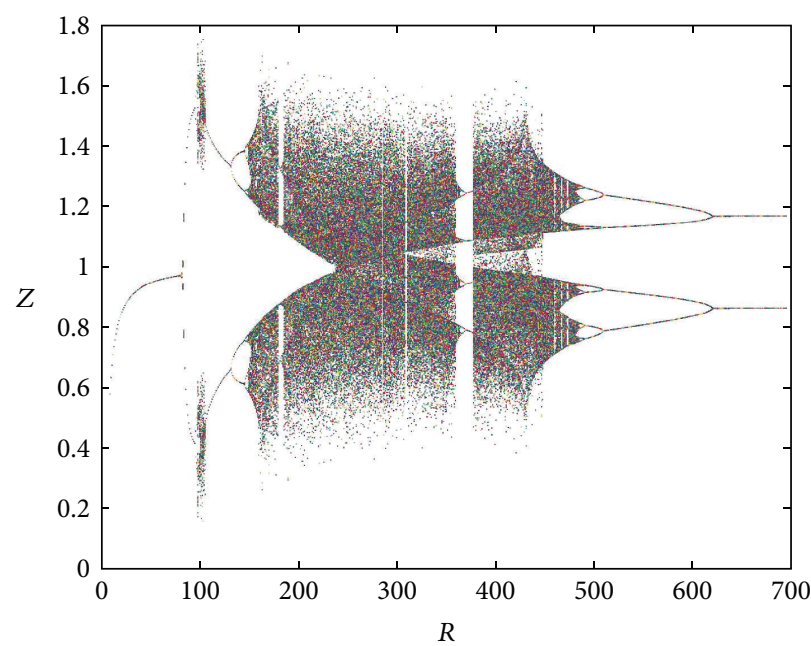

(b)

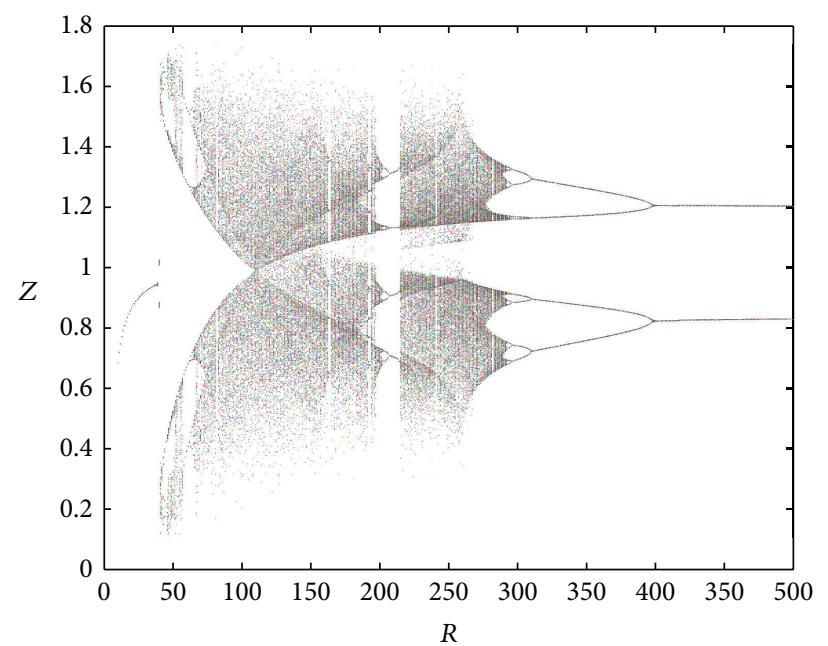

(c)

Figure 4: Bifurcation diagrams for (a) Le $=0.1, R_{s}=15$, (b) Le $=0.1, R_{s}=30$, and (c) Le $=0.4, R_{s}=15$.

in Figure 5(c). This corresponds to the first wide periodic window within the chaotic regime. In Figure 5(d) we can observe that the data points do align in such a way as to produce an almost clear projection of unconnected points on the projected plane. Increasing the value of $R$ further shows the dynamical behaviour's return to being chaotic again at $R=300$ as shown in Figure 5(e). At $R=360$ and $R=400$, we have a period- 8 and period- 4 periodic solutions as shown in Figures 5(f) and 5(g). Figure 5(h) shows that a period2 periodic solution takes over at $R=500$ and a period- 2 periodic type remains when the solutions at higher values of $R$ are obtained. We conclude the observation around these regimes of periodic windows within the broadband of chaotic solutions by pointing out a sequence of period-halving as one increases the Rayleigh number.

Figure 6 shows the projections of the trajectory's data points on the $X-Y-Z$ plane for Le $=0.1$ and $R_{s}=30$. The subcritical values for limit cycle and homoclinic explosion occur at $R_{c 2}=82.18$ and $R=98$ as shown in Figures 6(a) and 6(b), respectively, while period- 8 is observed at $R=364$ and 370 as presented in Figures 6(c) and 6(d). The dynamical behaviour returns to being chaotic again as $R$ increases; this happens at $R=400$ as shown in Figure 6(e). Figures 5(f) and $5(\mathrm{~g})$ show a period- 8 and period- 4 periodic solutions at $R=490$ and $R=500$. Figure $6(\mathrm{~h})$ shows that a period -2 periodic solution takes over at $R=600$ and remains the same behaviour when the solutions at higher values of $R$ are continued.

5.2. Lyapunov Exponents. The convergence plot of the Lyapunov spectrum for system (12) is shown in Figure 7. The alogrithm as proposed by [24] was employed for this purpose. The values of the Lyapunov exponents for system (12) are tabulated in Table 1. From these results, we can conclude that for eigenvalue $\lambda_{1}$ the system is always unstable and chaotic with the increasing $R$. For $\lambda_{2}$, the system alternates between being stable and dissipative to unstable and chaotic when the value of $R$ is increased. For eigenvalues $\lambda_{3}, \lambda_{4}$, and $\lambda_{5}$, the system is always stable and dissipative with the increasing $R$. 


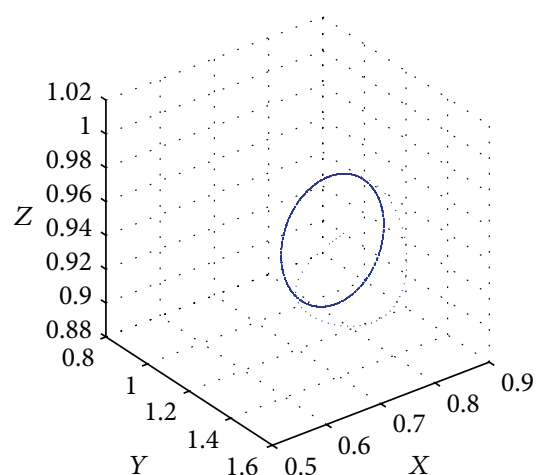

(a)

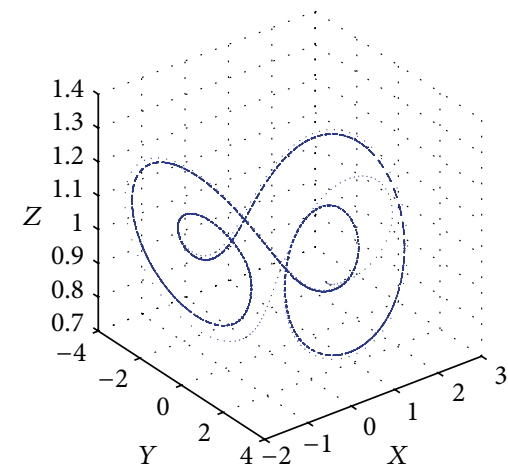

(d)

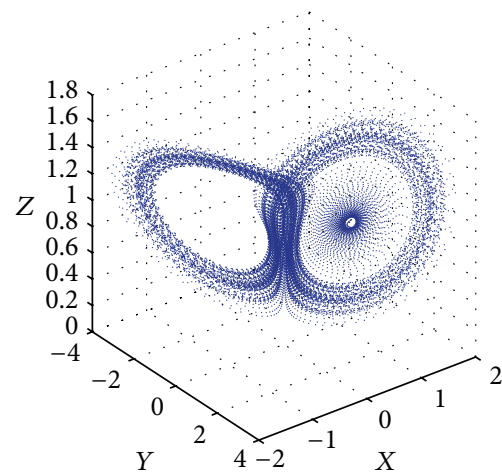

(b)

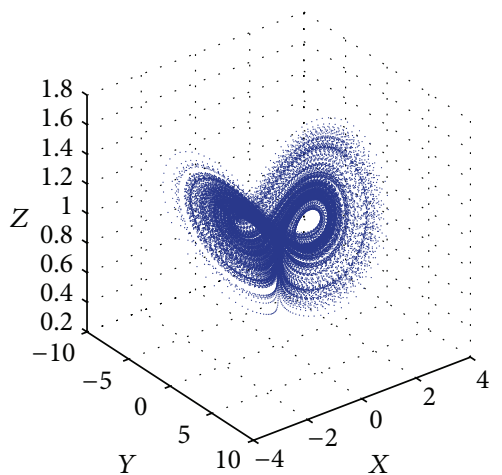

(e)

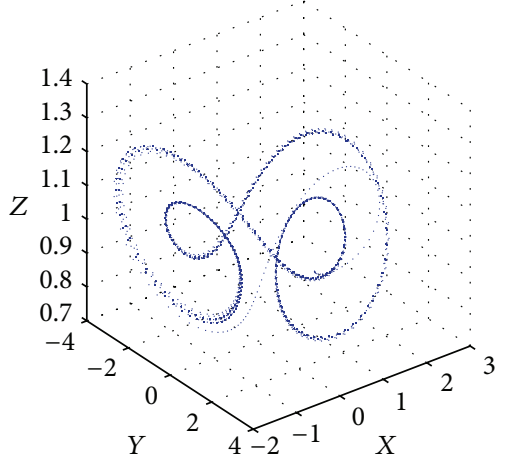

(c)

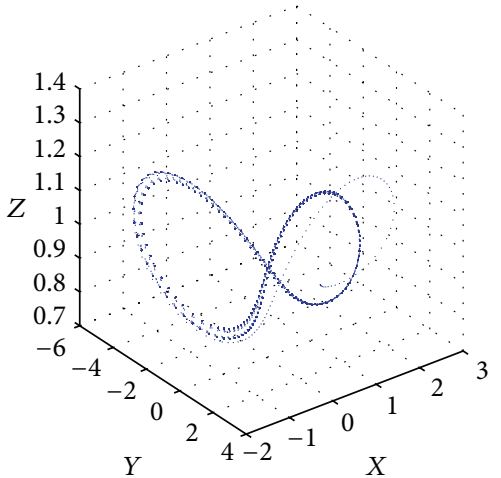

(f)

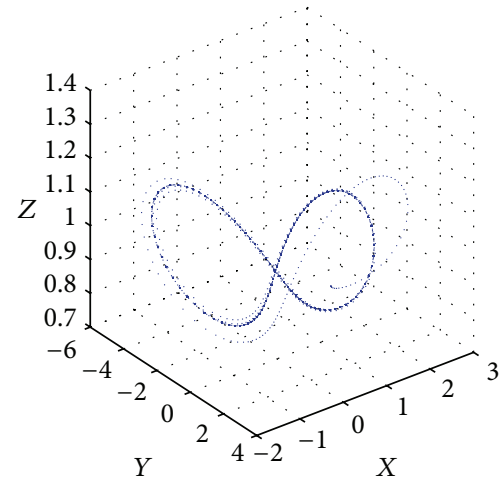

(g)

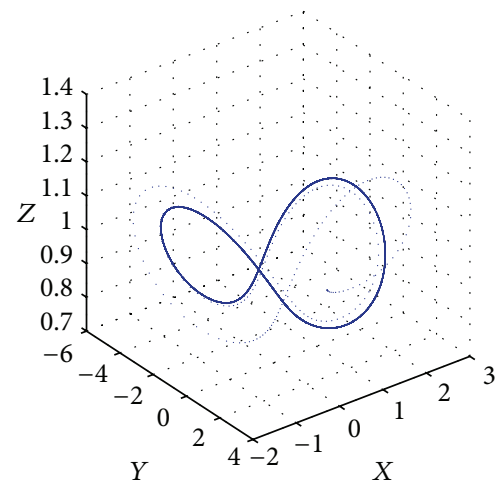

(h)

Figure 5: Phase portraits for (a) $R \simeq 46.37$, (b) $R=48$, (c) $R=250$, (d) $R=260$, (e) $R=300$, (f) $R=360$, (g) $R=400$, and (h) $R=500$ for the case where $\mathrm{Le}=0.1, R_{\mathrm{s}}=15$.

TABLE 1: Lyapunov exponents for system (12) computed from 10,000 data points for the case where Le $=0.1$ and $R_{s}=15$.

\begin{tabular}{lccccc}
\hline$R$ & $\lambda_{1}$ & $\lambda_{2}$ & $\lambda_{3}$ & $\lambda_{4}$ & $\lambda_{5}$ \\
\hline 46.37 & 2.674585 & -3.572664 & -7.683913 & -12.847196 & -28.904144 \\
48 & 2.612254 & -3.302684 & -7.921422 & -12.710783 & -29.010697 \\
260 & 6.350478 & 0.626358 & -4.509057 & -13.941471 & -38.856433 \\
300 & 7.469197 & -0.458284 & -4.653033 & -14.610687 & -38.076093 \\
360 & 8.589279 & 0.219833 & -4.754681 & -15.043590 & -39.338205 \\
400 & 8.404066 & -0.611687 & -4.530720 & -12.889258 & -40.698489 \\
500 & 4.451812 & 4.442320 & -3.196173 & -12.386501 & -43.632794 \\
\hline
\end{tabular}




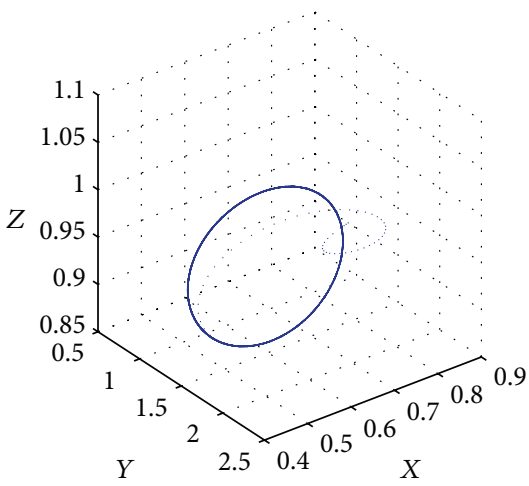

(a)

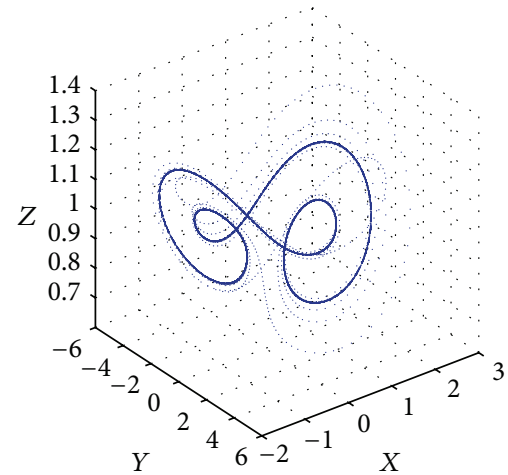

(d)

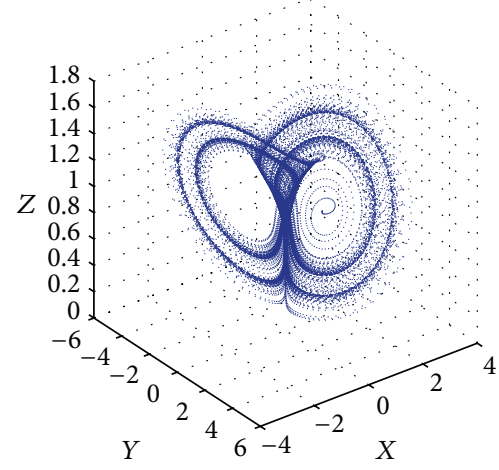

(b)

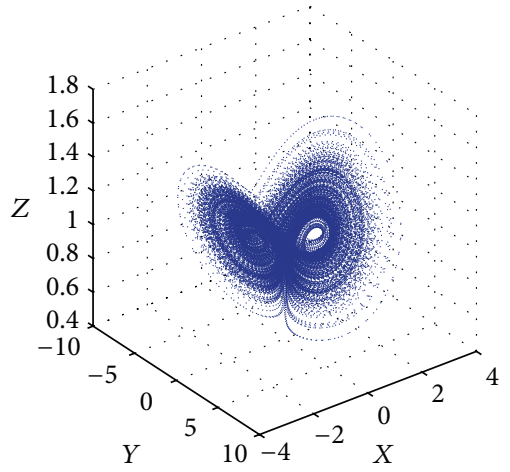

(e)

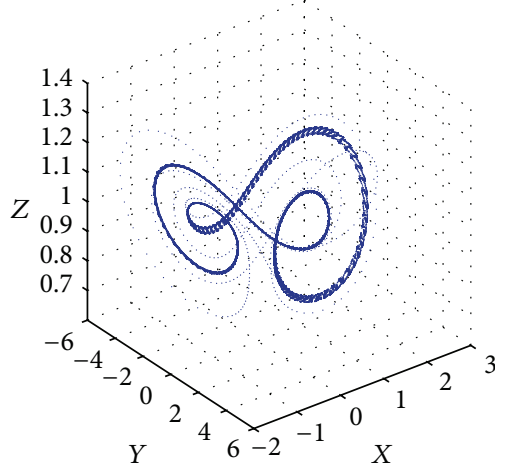

(c)

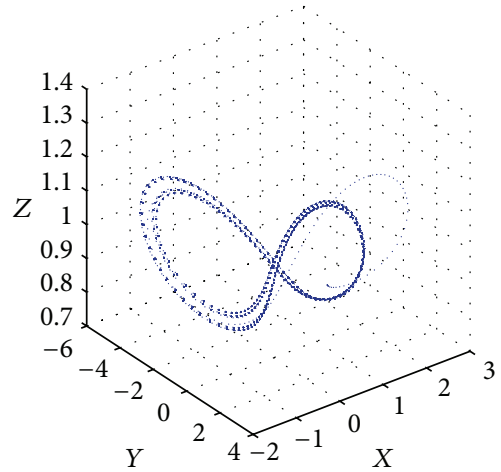

(f)

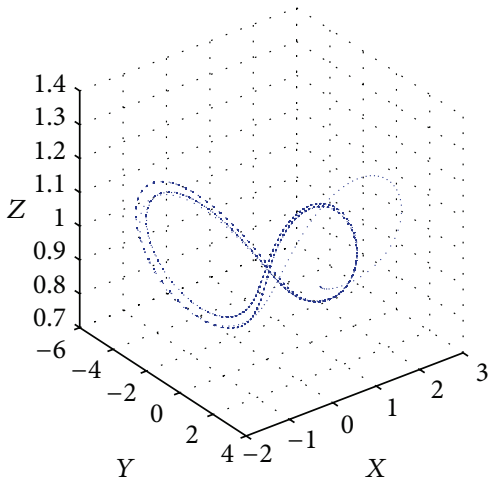

(g)

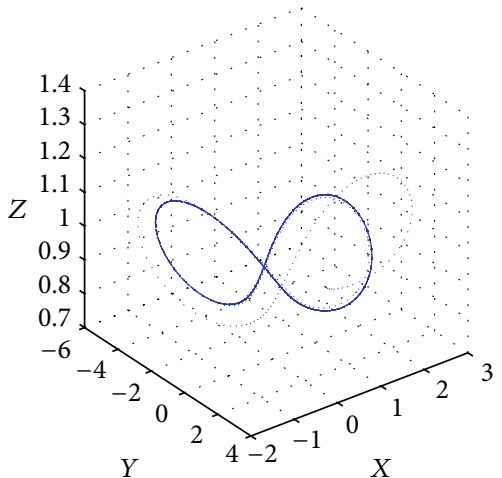

(h)

Figure 6: Phase portraits for (a) $R=82$, (b) $R=98$, (c) $R=364$, (d) $R=370$, (e) $R=400$, (f) $R=490$, (g) $R=500$, and (h) $R=600$ for the case where Le $=0.1, R_{s}=30$. 


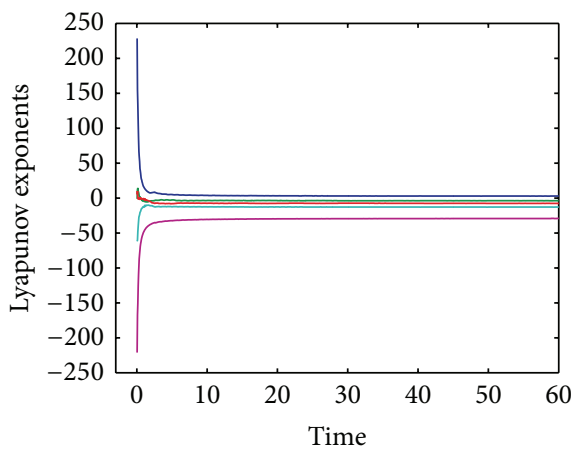

(a)

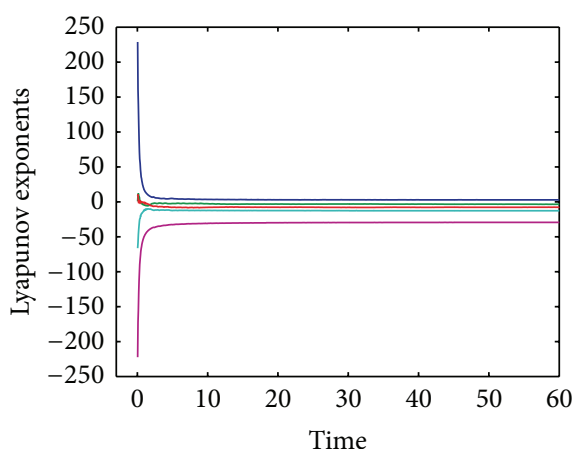

(c)

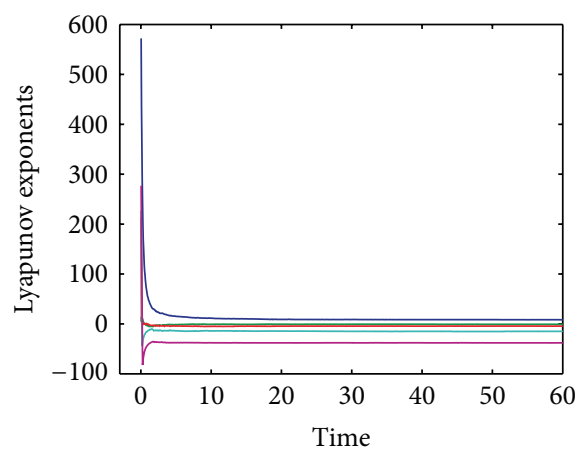

(e)

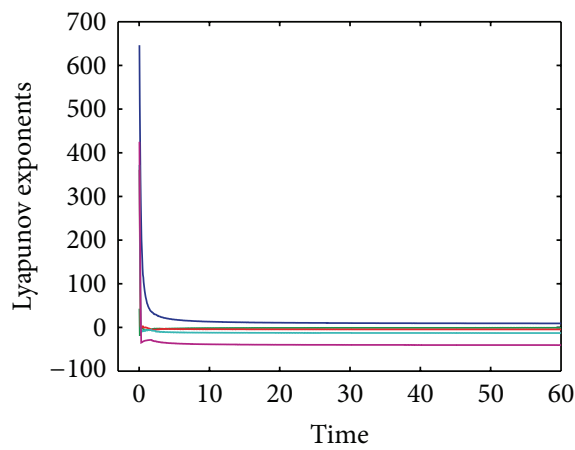

(g)

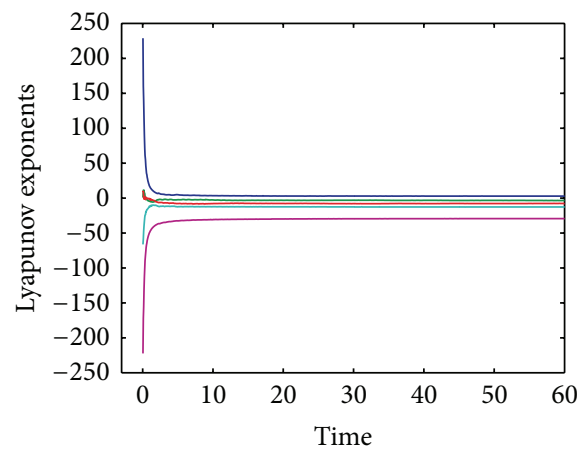

(b)

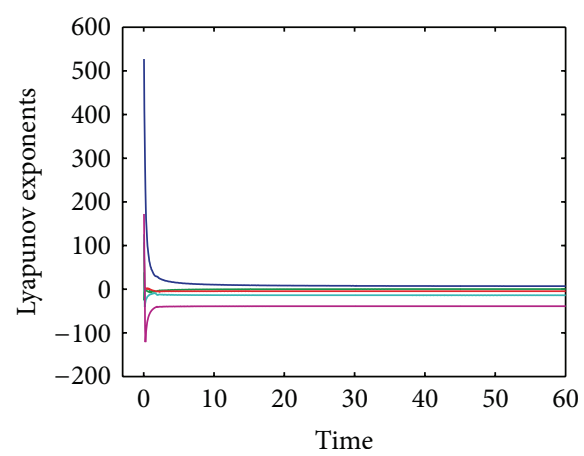

(d)

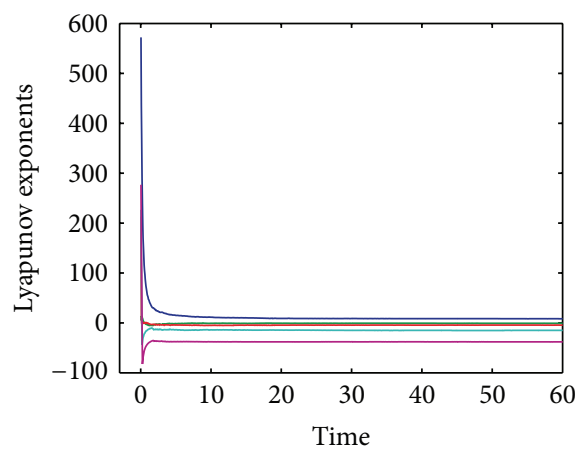

(f)

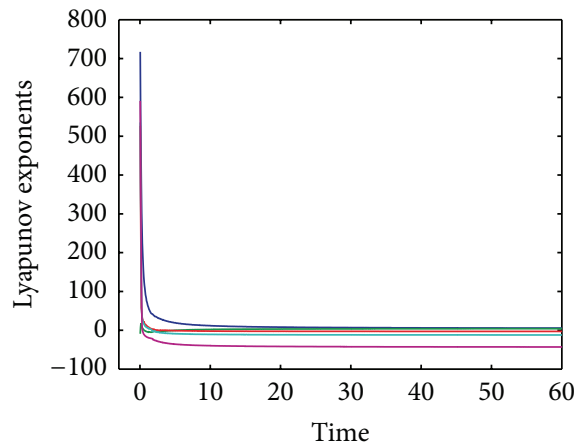

(h)

Figure 7: Dynamics of Lyapunov exponents for (a) $R=46.37$, (b) $R=48$, (c) $R=250$, (d) $R=260$, (e) $R=300$, (f) $R=360$, (g) $R=400$, and (h) $R=500$ for the case where $\mathrm{Le}=0.1, R_{s}=15$. 


\section{Conclusion}

In this work, chaotic behaviour in double-diffusive convection in a fluid layer has been investigated. A five-dimensional model of chaotic system was obtained using the Galerkin truncated approximation. The transition from steady convection to chaos via a Hopf bifurcation produced a limit cycle which may be associated with a homoclinic explosion at a slightly subcritical value of the thermal Rayleigh number. Both the solutal Rayleigh number and Lewis number affect the stability of the system. Increasing the Rayleigh number shows that the trajectory of the data points pointing out a sequence of period-halving and the behaviour remains the same at a higher Rayleigh number. The different transitions of the system, can be implied by the different values of the Lyapunov exponents. Negative eigenvalues lead to a stable and dissipative system and positive eigenvalues show that the system is always unstable and chaotic, while alternate eigenvalues suggest different transitions of the system (i.e., stable and dissipative to unstable and chaotic) as the value of the Rayleigh number is increased.

\section{Acknowledgments}

The authors acknowledge the financial support from Universiti Malaysia Terengganu (Grant no. TPM-68006/2012/49), Universiti Kebangsaan Malaysia (Grant no. DIP-2012-12), and University of Malaya (Grant no. RG172-11AFR).

\section{References}

[1] E. N. Lorenz, "Deterministic nonperiodic flow," Journal of the Atmospheric Sciences, vol. 20, pp. 130-141, 1963.

[2] J. Awrejcewicz, Bifurcation and Chaos in Coupled Oscillators, World Scientific, Singapore, 1991.

[3] J. Awrejcewicz and V. A. Krysko, Chaos in Structural Mechanics, Springer, Berlin, Germany, 2008.

[4] G. Qi, S. Du, G. Chen, Z. Chen, and Z. Yuan, "On a fourdimensional chaotic system," Chaos, Solitons and Fractals, vol. 23, no. 5, pp. 1671-1682, 2005.

[5] G. Chen and T. Ueta, "Yet another chaotic attractor," International Journal of Bifurcation and Chaos in Applied Sciences and Engineering, vol. 9, no. 7, pp. 1465-1466, 1999.

[6] W. B. Liu and G. A. Chen, "A new chaotic system and its generation," International Journal of Bifurcation and Chaos, vol. 13, pp. 261-267, 2003.

[7] J. H. Lü, G. Chen, D. Cheng, and S. Celikovsky, "Bridge the gap between the Lorenz system and the Chen system," International Journal of Bifurcation and Chaos in Applied Sciences and Engineering, vol. 12, no. 12, pp. 2917-2926, 2002.

[8] Z. M. Chen and W. G. Price, "On the relation between RayleighBénard convection and Lorenz system," Chaos, Solitons and Fractals, vol. 28, no. 2, pp. 571-578, 2006.

[9] Y. Ookouchi and T. Hada, "Chaotic convection in a simple system modified by differential heating," Journal of the Physical Society of Japan, vol. 66, pp. 369-378, 1997.

[10] P. Vadasz, "Local and global transitions to chaos and hysteresis in a porous layer heated from below," Transport in Porous Media, vol. 37, no. 2, pp. 213-245, 1999.
[11] P. Vadasz, "Subcritical transitions to chaos and hysteresis in a fluid layer heated from below," International Journal of Heat and Mass Transfer, vol. 43, pp. 705-724, 2000.

[12] R. Idris and I. Hashim, "Effects of a magnetic field on chaos for low Prandtl number convection in porous media," Nonlinear Dynamics, vol. 62, no. 4, pp. 905-917, 2010.

[13] M. N. Mahmud and I. Hashim, "Effects of a magnetic field on chaotic convection in fluid layer heated from below," International Communications in Heat and Mass Transfer, vol. 38, no. 4, pp. 481-486, 2011.

[14] J. M. Jawdat, I. Hashim, and S. Momani, "Dynamical system analysis of thermal convection in a horizontal layer of nanofluids heated from below," Mathematical Problems in Engineering, vol. 2012, Article ID 128943, 13 pages, 2012.

[15] H. E. Huppert and J. S. Turner, "Double-diffusive convection," Journal of Fluid Mechanics, vol. 106, pp. 299-329, 1981.

[16] E. Knobloch, D. R. Moore, J. Toomre, and N. O. Weiss, “Transitions to chaos in two-dimensional double-diffusive convection," Journal of Fluid Mechanics, vol. 166, pp. 409-448, 1986.

[17] J. K. Bhattacharjee, Convection and Chaos in Fluids, World Scientific, Singapore, 1987.

[18] G. Veronis, "Effect of a stabilizing gradient of solute on thermal convection," Journal of Fluid Mechanics, vol. 34, pp. 315-336, 1968.

[19] I. N. Sibgatullin, S. Ja. Gertsenstein, and N. R. Sibgatullin, "Some properties of two-dimensional stochastic regimes of doublediffusive convection in plane layer," Chaos, vol. 13, no. 4, pp. 1231-1241, 2003.

[20] Y. S. Li, Z. W. Chen, and J. M. Zhan, "Double-diffusive Marangoni convection in a rectangular cavity: transition to chaos," International Journal of Heat and Mass Transfer, vol. 53, pp. 5223-5231, 2010.

[21] J. Awrejcewicz, V. A. Krysko, I. V. Papkova, and A. V. Krysko, "Routes to chaos in continuous mechanical systems. Part 1: mathematical models and solution method," Chaos, Solitons and Fractals, vol. 45, pp. 687-708, 2012.

[22] V. A. Krysko, J. Awrejcewicz, I. V. Papkova, and A. V. Krysko, "Routes to chaos in continuous mechanical systems. Part 2: modelling transitions from regular to chaotic dynamics," Chaos, Solitons and Fractals, vol. 45, pp. 709-720, 2012.

[23] J. Awrejcewicz, A. A. Krysko, I. V. Papkova, and A. V. Krysko, "Routes to chaos in continuous mechanical systems. Part 3: the Lyapunov exponents, hyper, hyperhyper and spatial-temporal chaos," Chaos, Solitons and Fractals, vol. 45, pp. 721-736, 2012.

[24] A. Wolf, J. B. Swift, H. L. Swinney, and J. A. Vastano, "Determining Lyapunov exponents from a time series," Physica D. Nonlinear Phenomena, vol. 16, no. 3, pp. 285-317, 1985. 


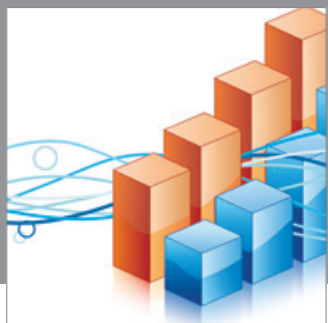

Advances in

Operations Research

mansans

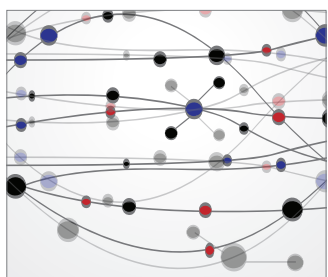

The Scientific World Journal
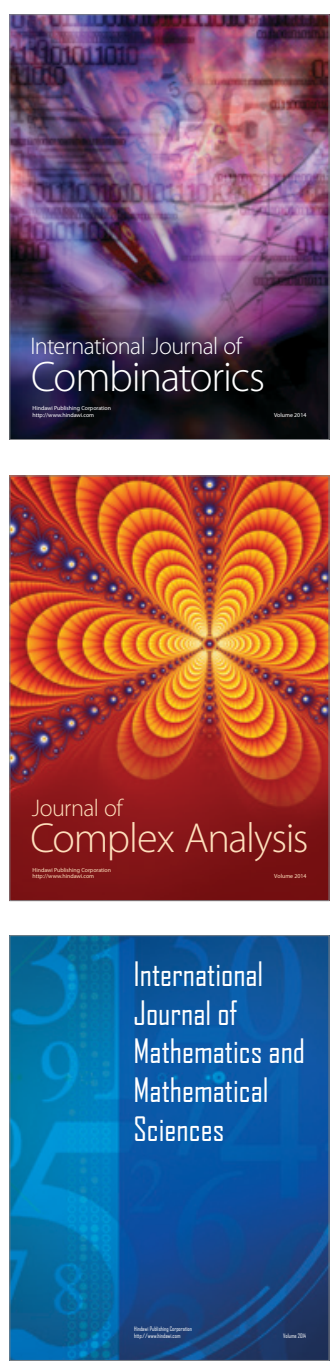
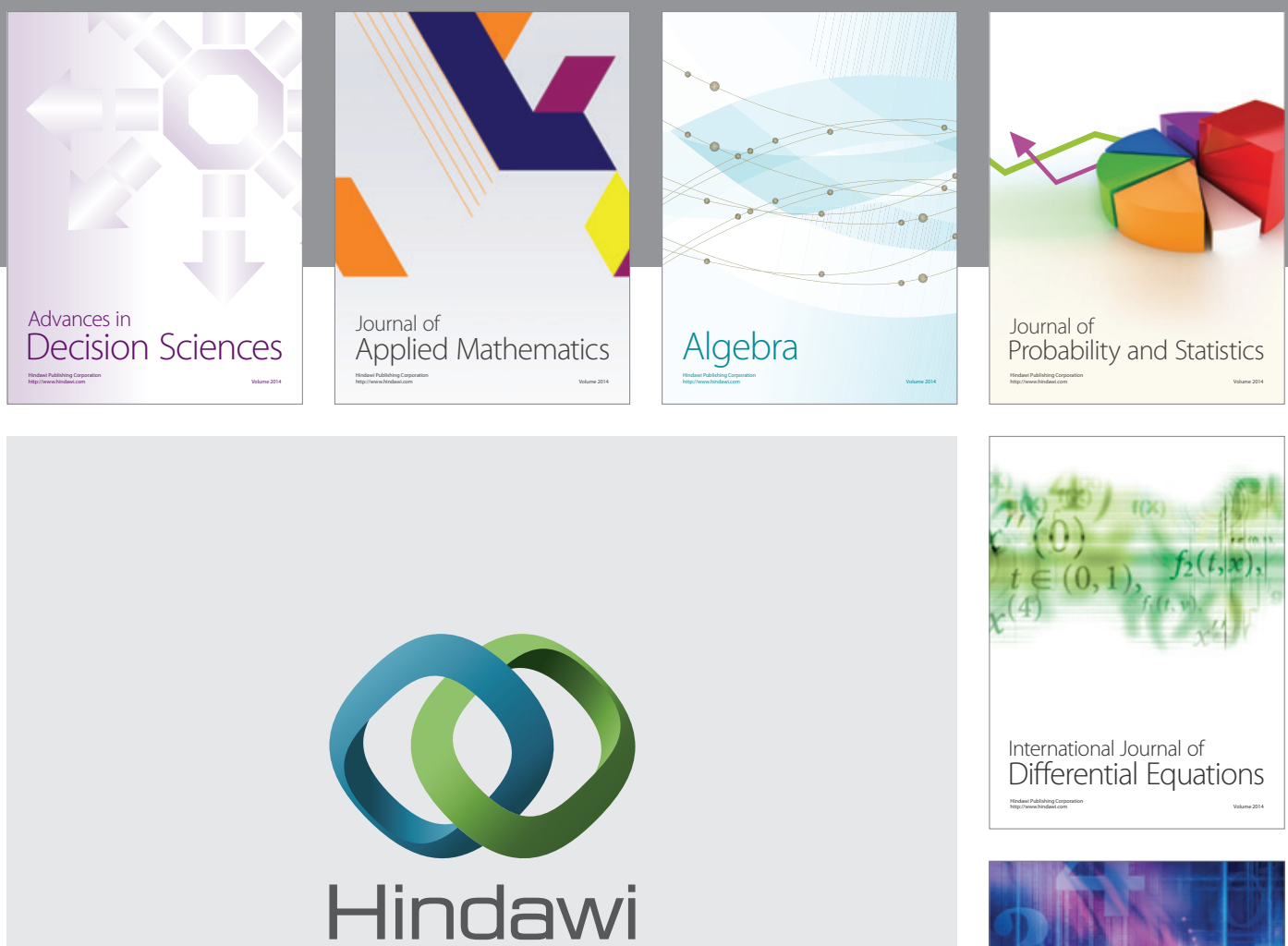

Submit your manuscripts at http://www.hindawi.com
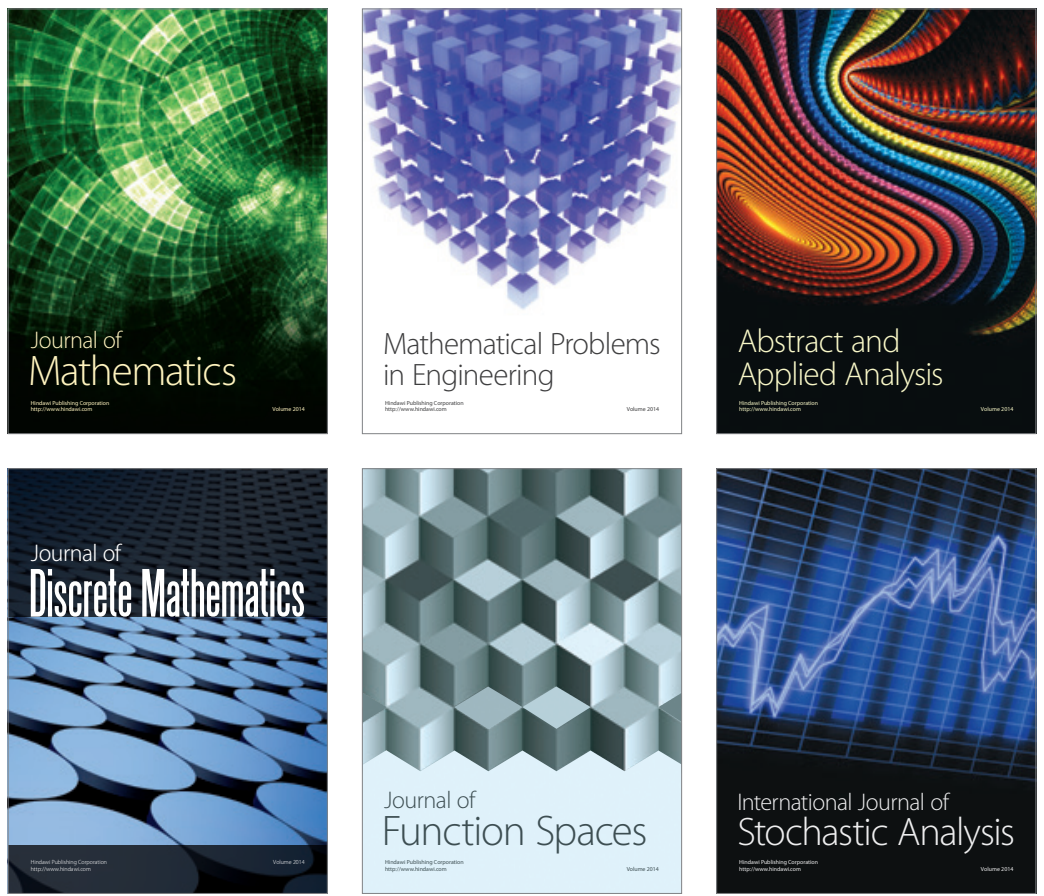

Journal of

Function Spaces

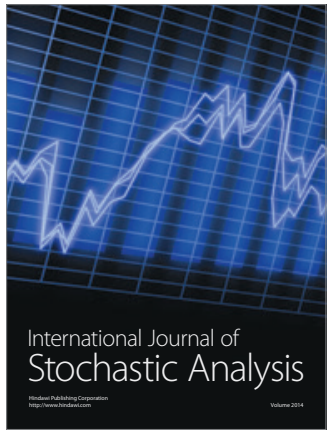

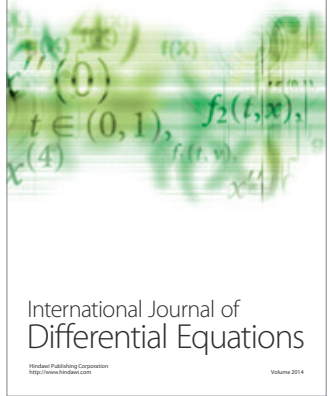
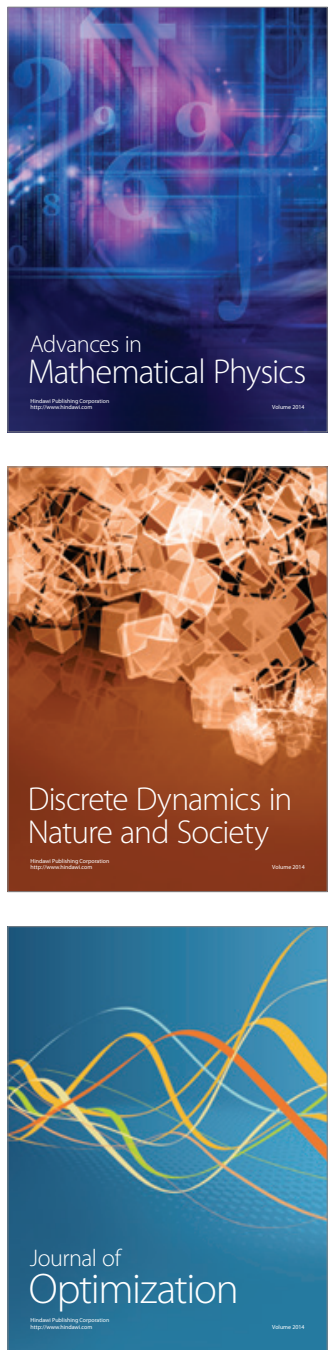